\title{
Applicability of Droop Regulation Technique in Microgrid - A Survey
}

\author{
Chitra Natesan ${ }^{1,2, a}$, Senthilkumar Ajithan ${ }^{3, b}$, Shobana Mani ${ }^{4, c}$, \\ and Prabaakaran Kandhasamy ${ }^{4, \mathrm{~d}}$ \\ 1 Anna University, Kotturpuram, Chennai, Tamil Nadu 600025, India \\ 2 Department of Electrical and Electronics Engineering, SKP Engineering College, Tiruvannamalai-606611, \\ India \\ 3 Velammal Engineering College, Velammal Nagar, Chennai, 600066, India \\ 4 SKP Engineering College, Tiruvannamalai-606611, India \\ E-mail: asivakumar.poruran@gmail.com (Corresponding author), bvastham@gmail.com, \\ cshoby45@gmail.com, dprabaakaran031@gmail.com
}

\begin{abstract}
Currently, the worth of power generation on the basis of renewable sources is rapidly growing. Correspondingly the microgrids and the DG units are impressed the researchers for their peculiar features. Power sharing is the major concern when various DGs are connected to the microgrid via power electronic converters. It is mandatory to achieve an appropriate power sharing when the manifold DGs are activated in parallel. For that, the two ultimate quantities-power angle $\delta$ and voltage magnitude $\mathrm{V}$ are regulated to acquire the real and reactive power sharing correspondingly. Many innovative control techniques have been used for load sharing. The most common method of local load sharing is the droop characteristics. Subsequently, there is a swift momentum in the advancement of researchers to meet the challenges of the droop control techniques in the power sharing concerns, an extensive literature review on active and reactive power sharing, voltage and frequency control in microgrid has been emphasized. The various conventional and modified droop control techniques/strategies that relates to power sharing issues have been highlighted in this work.
\end{abstract}

Keywords: Microgrid, distributed generation, power sharing, droop control.

ENGINEERING JOURNAL Volume 18 Issue 3

Received 21 November 2013

Accepted 6 March 2014

Published 10 July 2014

Online at http://www.engj.org/

DOI:10.4186/ej.2014.18.3.23 


\section{Introduction}

Contemporarily, the power sector is swapped to an epoch where the power exigency is accomplished by the distributed generation (DGs). The custom of DG eases the feeder loss, the reliability is enhanced and also its overall efficiency is enriched through the custom of unwanted heat [1]. Wind power, solar power and hydro power are the several renewable micro sources which are linked to DGs. Among these microsources wind power and solar power are considered to be most suitable economically during isolated microgrid [42]. As DGs are sustainable, it can be positioned very near to end-users [2].

In microgrid perception, it is a small scale distribution network and it is manipulated independently. Besides, Microgrid includes various components such as converters, DG units and storagable devices which are coupled with macrogrid [3].

The latest innovations such as solar cells and fuel cells (FC) are in desperate need of inverter in order to interrelate with the distribution system [4]. Therefore to afford a common load, an arrangement of VSC (voltage source converter) interfaced with microgrid is operated in parallel [5].

Fundamentally, a microgrid can be functioned in two operating environments. When the microgrid is in contact with the macro-grid at the junction of PCC (point of common coupling), then it is in gridconnected mode. When the microgrid is detached from the macro grid, then it is in island mode.

Even if any malfunction happens in microgrid it will switch automatically to island mode, so there won't be any interruption in the supply to the local area [6].

There are many practical concerns associated with microgrid operation such as interconnection patterns among microgrids and the main grid [7]; voltage-control strategies within a microgrid [8]-[10]; and frequency control during islanded operation. This paper surveyed on appropriate power sharing and frequency-control schemes for optimal performance of a microgrid with manifold DGs. The reason to concentrate on optimum power sharing is, for the reliable operation of microgrid to optimize the voltage profile and also to reduce losses. Appropriate power sharing in island mode can be acquired only by means of control schemes. Among various control schemes, this paper highlighted on droop control techniques. The droop control types such as conventional droop control (P-F droop control \& Q-V droop control) and modified conventional droop control (P-V \& Q-F droop control, V-F droop control and Angle droop) are spotted in this work.

The primary focus of droop control is to retain the fundamental frequency \& the voltage magnitude of microgrid with manifold DGs in autonomous mode so that the appropriate powers are shared [11]. Frequently used droop control technique to enhance power sharing and the voltage/ frequency synchronization are real power-frequency $(\mathrm{P}-\mathrm{F})$ droop control and reactive power-voltage magnitude $(\mathrm{Q}-$ V) droop control. Consequently its implementation is simple and it empowers decentralized control of manifold distributed generations (DGs) [12].

The organization of this paper is as follows. The droop control, strategy is briefly explained in section 2 . In section-3 various conventional droop control methods are discussed for proper power sharing. Section-4 presents modified conventional droop control methods for reliable power sharing. Here the conventional parameters are modified and variant methods are proposed. Section- 4 describes the wireless sensor network based power sharing.

\section{Droop Control Theory}

In microgrid, the system reliability and stability is achieved only by the voltage regulation when more micro sources are interconnected. This voltage regulation damps the reactive power oscillations and voltage. In a complex power system, when multiple DGs are attached to the microgrid, the power sharing among them is made properly with the help of a control strategy called droop control. Droop control also enables the system to disconnect smoothly and reconnect routinely to the complex power system [43].

The role of droop control in power sharing is that it control the real power on the basis of frequency droop control and it controls the reactive power on the basis of voltage control [44]. The voltage and frequency can be manipulated by regulating the real and reactive power of the system. This forms a conventional droop control equation.

In a transmission line, the real and reactive power are designed as:

$$
\mathrm{P}=\frac{\mathrm{V}_{1} \mathrm{~V}_{2}}{\mathrm{X}} \sin \delta
$$




$$
\mathrm{Q}=\frac{\mathrm{V}_{1}^{2}}{\mathrm{X}}-\frac{\mathrm{V}_{1} \mathrm{~V}_{2}}{\mathrm{X}} \cos \delta
$$

In the above mentioned equation (1) and (2), Resistance (R) is neglected for an overhead transmission lines as it is much lower than inductance $(\mathrm{L})$. Also the power angle $\delta$ is lesser, Therefore, $\sin \delta=\delta$ and $\cos$ $\delta=1$.

$$
\begin{gathered}
\delta=\frac{\mathrm{XP}}{\mathrm{V}_{1} \mathrm{~V}_{2}} \\
\mathrm{~V}_{1}-\mathrm{V}_{2} \cong \frac{\mathrm{XQ}}{\mathrm{V}_{1}}
\end{gathered}
$$

Hence from the above equation (3) and (4), it is clear that the power angle $\delta$ can be controlled by regulating real power P. Also the voltage V1 can be controlled through reactive power Q. dynamically, the frequency control leads to regulate the power angle and this in turn controls the real power flow [45]. Finally, the frequency and voltage amplitude of the microgrid are manipulated by adjusting the real and reactive power autonomously. As a result, the frequency and voltage droop regulation can be determined as:

$$
\begin{aligned}
\mathrm{f}-\mathrm{f}_{0} & =\mathrm{K}_{\mathrm{p}}\left(\mathrm{P}-\mathrm{P}_{0}\right) \\
\mathrm{V}-\mathrm{V}_{0} & =\mathrm{K}_{\mathrm{q}}\left(\mathrm{Q}-\mathrm{Q}_{0}\right)
\end{aligned}
$$

The relationship between real power - frequency and reactive power - voltage can be manipulated from the equations (5) and (6):

$$
\begin{gathered}
\mathrm{f}=\mathrm{f}_{0}+\mathrm{K}_{\mathrm{p}}\left(\mathrm{P}-\mathrm{P}_{0}\right) \\
\mathrm{V}=\mathrm{V}_{0}+\mathrm{K}_{\mathrm{q}}\left(\mathrm{Q}-\mathrm{Q}_{0}\right)
\end{gathered}
$$

where $\mathrm{f}, \mathrm{V}=$ The frequency and voltage at a new operating point; $\mathrm{P}, \mathrm{Q}=$ Active and reactive power at a new operating point; $\mathrm{f}_{0}, \mathrm{~V}_{0}=$ Base frequency and voltage; $\mathrm{P}_{0}, \mathrm{Q}_{0}=$ Temporary set points for the real and reactive power; $\mathrm{K}_{\mathrm{p}}, \mathrm{K}_{\mathrm{q}}=$ Droop constant.

\subsection{Droop Control Block Diagram}

The droop control theory is explained with the block diagram as shown in Figure 1. This droop control block is composed of two function blocks:

(1) Frequency droop control

(2) Output limit control.

The central controller delivers the inputs such as the system frequency (f) and the output of power generation (P), or feeder flow (FL) and set points.

Inputs are local measurements of frequency $(\mathrm{f})$ and power output $(\mathrm{P})$, or feeder flow (FL), and the set points are provided by the central controller. The output of the current controller is current reference signal of the d-axis [13].

In power grid attached mode, the microgrid frequency is same as the rated value, so that the power output $(\mathrm{P})$ and the feeder flow (FL) are sustained to the fixed value. When the microgrid is detached from the power grid, the power mismatch are balanced by the droop control automatically. With this the system attain its steady state and finally the system frequency is restored to its rated value. 


\section{Droop control}

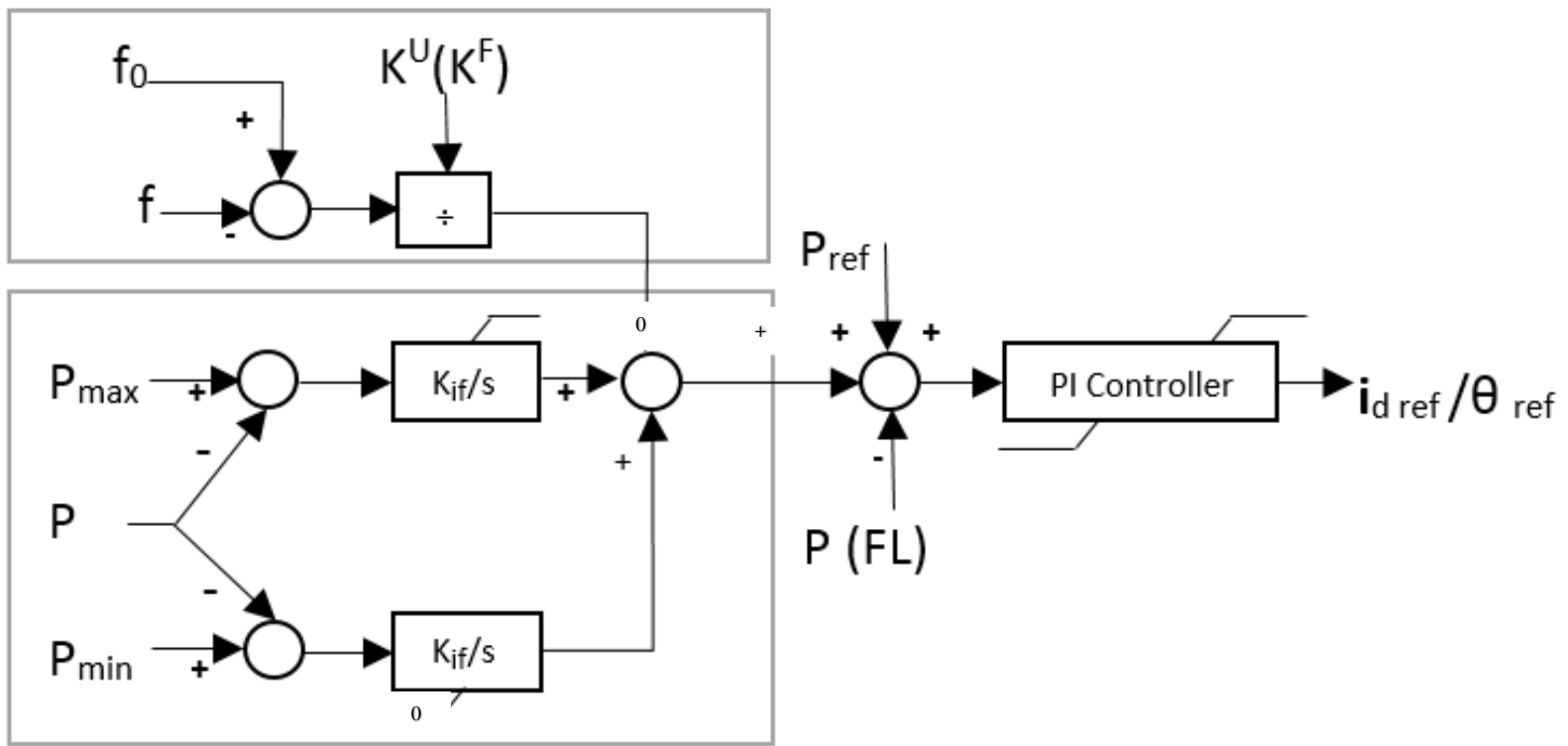

\section{Output limit control}

Fig. 1. The Droop control block diagram.

\subsection{Types of Droop Control}

Droop control is categorized on the basis of regulating the system parameters. They are:

(1) Conventional droop control

This is a common type of droop control. Conventionally, the system frequency and the voltage amplitude are regulated with respect to the real and reactive power generation of the system. Hence forth the power sharing in microgrid is attained by the output power generation according to its DG's power rating. The conventional droop control is further classified as:

i. $\quad$ Real power-frequency $(\mathrm{P}-\mathrm{F})$ droop control

ii. Reactive power-voltage magnitude $(Q-V)$ droop control

(2) Modified droop control

In the case of conventional droop control, the resistance is taken as negligible quantity because $\mathrm{L}>>>$ $\mathrm{R}$ in high voltage lines. Whereas this assumption is not suitable for microgrid as it operates at low and medium voltage. Hence modified droop control arise by changing the parameters of the conventional droop control as per their system requirement. The modified droop control referred in this work are

i. $\quad$ P-V and Q-F droop control

ii. $\quad$ V-F droop control

iii. Adaptive transient droop

iv. SoC (State Of Charge) based droop control

v. Angle droop control

vi. Q-V dot droop control

\section{Power Sharing Method Using Conventional Droop Control}

In this segment, power sharing in a microgrid was explored using FL-F (feeder flow - frequency); P-F and Q-V droop characteristics.

Seon ju and Khanh-Loc Nguyen et al. [13], [2] discoursed about the power sharing approaches by considering UPC (unit power output control) \& FFC (feeder flow control) control modes with manifold DGs. 
In [13], the authors emphasized the active power control of DGs under two different options 1) UPC (P-F droop control) 2) FFC (FL-F droop control). In accordance with the reference of power, the DG output power is controlled regularly in UPC mode. Whereas the feeder power flow is employed with respect to the reference of flow in FFC mode. The performance of the system was scrutinized on the basis of power control modes under several circumstances in order to confirm appropriate power sharing. The different system circumstances were as follows:

(1) When load variation occurs during grid-attached mode, the nominal value of the frequency was maintained by the main grid. Also the feeder flow was controlled constantly in the FFC mode.

(2) When load variation occurs in island operation, the frequency changes in proportion to the load variation in UPC mode. Whereas, in FFC mode the frequency sustained constantly.

(3) When the microgrid was disconnected from the main grid, the power flows fallen to zero. In the course of transition operation, power was not accurately shared in the FFC mode of series configuration. So as to mitigate this drawback, droop constant of FFC mode is modified. Therefore a new droop control is designed for FFC or a combination of FFC with UPC in series configuration. This results in accurate power sharing. As a final point, the author concluded that the capacity of power flowing between the microgrids could be forecasted and regulated by the FFC mode. Therefore FFC mode is the most beneficial for power system workers because it compensates the load variation.

Seon ju extended his research [2] by employing the same approach as in [13] by evaluating constant load regulation in grid-tied mode and frequency deviation minimization in transition mode. In the course of transition mode with FL-F droop control, the microgrid was stabilized and the load was shared among the DGs properly. This was done by shifting the control modes among UPC and FFC. Here, FL reference value was fixed, so that a constant load was achieved by the microgrid. The output of DGs was maximized to minimize FL at the peak load. So this leads to frequency deviation minimization in transition mode. Finally, the DGs power control mode must always be in FFC mode, so that it maintains the power exchange unaltered among microgrids and main grid.

\subsection{Active and Reactive Power Sharing}

This section emulates an outline of the active and reactive power sharing through conventional droop control.

The power sharing technique along with stability enhancement with P-F and Q-V droop control was deliberated by Jinwei He and Ritwik Majumder, [14], [16]. They concentrated on appropriate power sharing in the island operation of microgrid.

An appropriate power sharing that suits even for a complex configuration is exhaustively described in [14]. By reducing the errors of real-reactive power, the power was shared properly. In order to detect the error and for proper power sharing, this method introduces a small real-reactive power disturbance that was triggered by the low bandwidth signal from the central controller. This work is comprehended over two phases viz, (i) Before compensation: initial powers are shared by conventional droop control. The $\mathrm{P}_{\text {avg }}$ was measured in this stage and it is used in the next stage. (ii) After compensation: the inclusion of real-reactive power transient compensates the error in power sharing and this phase automatically switches over to the first phase.

However, in the view of Ritwik Majumder [16], appropriate power sharing was accomplished by means of high angle droop gains. But the use of high angle droop gain has an adverse effect on overall stability. So, to overcome this adverse effect, it is necessary to design a supplementary droop around a conventional droop control for each DG unit. By the state space model analysis, microgrid stability through high angle droop gain is examined. In island operation, the converter interfaced DGs are influenced by the network dynamics because of its rapid switching action. Therefore, for the purpose of stability examination differential equation suits for network model. Hence, this confirms suitable load sharing particularly in weak system conditions. In along with the suitable load sharing, the supplementary loop was also used to stabilize the system within operating condition's range.

Another interesting technique was categorized by F. Katiraei [18], a small-signal dynamic model of a manifold DG was designed for Eigen studies as well as active and reactive power management strategies (PMS). Conventional and electronically interconnected DG units and the network were characterized by this model. It also accounts for the frequency deviance when microgrid is in island operation. Finally, the system performance was enhanced by the Eigen analysis under each PMS. The two parts of PMS include: 
(1) The active power generation block: It determines the real power output at the point of common coupling (PCC) depended on frequency deviations of the DG.

(2) The reactive power regulator block: This block controls the voltage of the equivalent DG-bus in addition the reactive power of the load was also compensated at the terminal.

The blocks of the PMS is explained with the two subsections:

(1) Active power control approaches is based on

i. Droop characteristics of frequency

ii. Complimentary frequency refurbishment scheme.

(2) Reactive power control approaches is based on

i. Droop characteristic of voltage

ii. Regulation of voltage

iii. Compensation of reactive power.

The result of these strategies reveals that the PMS enhances the system performance through the designed dynamic small-signal model.

Conversely, Farzad Razavi [20] focused on optimization of droop controller in MATLAB/ SIMULINK using Genetic Algorithm (GA). The droop coefficient is chosen randomly in such a way that it reduces the frequency deviation. Further the droop coefficients are optimized by means of Genetic algorithm, which effectively curtail the optimum frequency deviance. Also in this process of optimization, the load is delivered by the DGs. The droop coefficients are chosen same and the power is shared equally for a step change in the load. Along with the optimization of droop coefficients and power sharing, this work also focuses on the stability analysis. In order to confirm the good performance of the controller and to accomplish the system stability, the current and voltage controller PI gains were enhanced by GA. The simulation result are proven under two cases:

In case (i) - the droop coefficient are chosen on the basis of the equation (9) and (10)

$$
\begin{aligned}
& \mathrm{m}=\frac{\Delta \mathrm{W}}{\mathrm{P}_{\text {max }}} \\
& \mathrm{n}=\frac{\Delta \mathrm{V}}{\mathrm{Q}_{\text {max }}}
\end{aligned}
$$

where $\mathrm{m}=$ droop of coefficient of active power; $\mathrm{n}=$ droop coefficient of reactive power; $\Delta \mathrm{w}=$ maximum permissible deviation of frequency; $\Delta \mathrm{V}=$ maximum permissible deviation of voltage.

From this obtained droop coefficients, the frequency of the microgrid is manipulated. Even though the obtained frequency is within the permissible limit, it can be reduced further by optimizing the droop coefficient using GA, which is shown in case (ii).

In case (ii) - the droop coefficients were chosen based the constraints laid by the GA. With this the frequency deviation becomes much smaller than case (i) and the system performance is enhanced.

Finally, it was concluded that the system performance was better by means of the improved PI gains. Furthermore frequency deviation was reduced through the enhancement of PI gains and droop coefficient.

Farhad Shahnia [15] tried a system with nonlinear and unbalanced loads by considering the power quality improvement as well as power sharing. In this work, the microgrid arrangement resides four DGs viz. they are diesel generator, photovoltaic array, fuel cell and a storage battery. The three DGs (FC, PV and battery) are interfaced with converters whereas the diesel generator is an inertial. Here, the DG which is closer to the non-linear and unbalanced load is chosen as a compensator for power quality enhancement. The power was shared by the remaining DGs proportionate to their rating. The distributed power sharing between the DGs were accomplished by the custom of conventional droop control. The compensator (DG) works in two modes according to the non-linear load power requirement.

Mode1: In this mode, the non-linear load power demand is less than the compensator (DG) power rating. So, the nonlinear load demand and portion of remaining load's power demand is supplied by the compensator. This leads to injection of power towards the microgrid by means of the compensator. Hence this shows that the real-reactive power and the current of microgrid are negative. In this mode, when the power demand is amplified above the rated value of compensator, this mode will be switched to the mode 2 by the action of control scheme.

Mode2: In this mode, the non-linear load power demand is greater than the compensator (DG) power rating. The compensator supplies only a portion of nonlinear load demand, while the remaining portion of the power demand is satisfied by the microgrid by means of droop control. In this case, the real-reactive 
power and the current of microgrid are likely to be positive. On both the means, power was shared proportionally based on their ratings by the microsources with the help of droop control. Hence, the compensator not only compensates the system non-linearity but also it is involved in power sharing among the microsources.

Johannes Schiera [19] did his research in the perception of stability analysis for a mesh configuration of microgrid interfaced with inverter via P-F \& Q-V droop control in an island mode. The voltage and frequency stability may affect in different cases viz., during load variation, during mode transitions or in faulty cases. Hence in this work, the stability of the microgrid in course of power sharing using droop control is also focused. A condition is derived by a port Hamiltonian description in order to ensure the stability. This also confirms the stability of inverter-interfaced microgrid which was achieved through common interconnected topology and adjustable frequencies along with voltage amplitudes of the inverter model. They also considered the constraint in the stability of voltage and frequency in a microgrid during power sharing among the DGs.

\subsection{Active Power Sharing}

This section describes how the active power is shared among the DGs in an island microgrid via P-F \& QV droop control.

In the area of DGs with Low Voltage, Michaela Griffiths, Colin Coates et al. [6] designed a modeling and explored its performance by means of P-F \& Q-V droop control. They also motivated on testing the controllers which were suggested for power sharing among DGs. In this work Q-E droop control, various tests are performed to prove the effectiveness of Q-E droop control. They are:

i. Reactive current circulation: It generates more real power, reactive power absorption are reduced significantly.

ii. Unbalanced loads: The resistive load is converted from dq frame by means of custom block.

In this work, droop control is used to control the inverters so that the load voltage and the power were regulated by controlling the terminal voltage and frequency. The control algorithm includes voltage control and power control. By governing the voltage magnitude and voltage angle of the inverter, voltage regulation and power sharing is realized. The constraints in voltage control are the error in the set points of voltage which tends to circulate reactive power among the inverters. In order to lessen this problem, Q-E droop is used. The power controller normalizes the power angle via PI control driven by P-F droop compensator. Finally, by modifying the inverter's power angle, the power is shared among them. Therefore, power sharing by inverters was permitted by droop control and the complete modelling was implemented in the reference of dq frame.

\subsection{Reactive Power Sharing}

Appropriate Reactive power sharing among the manifold DGs during standalone mode by way of P-F \& $\mathrm{Q}-\mathrm{V}$ droop control was revealed in this division.

A new dimension of approach was conferred by Qobad Shafiee and A. Micallef et al. [21], [25] about the secondary control for reactive power sharing in island operation with P-F \& Q-V droop control. In this pattern a secondary control loop was instrumented, which is an external loop requiring a low bandwidth communications link. This loop was implemented in a central controller to attain equal reactive power sharing among the inverters.

In [21], Networked Control Systems Methodology was employed to refurbish voltage magnitude and frequency for satisfactory reactive power sharing in the microgrids. The control approach projected in this work reviews voltage and frequency control. (i) Frequency control: The frequency is restored by manipulating the frequency error of each DG and sent them to the other DGs whose measured frequency are averaged (ii) voltage control: Voltage deviation is compensated by measuring the voltage error. In this practice the failure of the sole unit would not affect the other unit, this is because of the distributed secondary control was independent from a central control. This control strategy proves a good behaviour in reducing the steady state errors of the voltage and frequency so that this involved in appropriate reactive power sharing among DGs.

In the same way given in [21], A. Micallef [25] projected the effects owing to mismatches in the line impedance between the microgrid and inverters in the island operation. The core idea of this work was to attain perfect distribution of the reactive power among the inverters even in the presence of mismatches in 
the power line. This was accomplished through the MGCC (microgrid central controller) communication which has a secondary loop around the controller. The MGCC gets the signal about the reactive power supplied to the microgrid that is provided by the microsources with the help of low bandwidth interaction. Then the MGCC controls the reactive power supplied by the inverter by means of an external loop. By the existence of the secondary loop, communication delays may occur between the microgrids and the central controller. But this never disturbs the reactive power sharing between them. The viability of the projected method was proven in accomplishing the reactive power sharing among the inverters coupled with the microgrid.

The concept of reactive power sharing with conventional droop control in an island microgrid was conferred by M. Kohansal as well as Jinwei He [17], [23]. They also concentrated on voltage \& frequency stability along with proper sharing of reactive power. Though their perception was similar but they differ from their control strategy.

M. Kohansal [17], found that the stability of voltage and frequency were highly influenced by the presence of several control characteristics of the elements like droop coefficient in VSI. In order to overcome this effect, an islanded microgrid is formed by modelling inverters, network, and loads individually. The Eigen values from the derived model is used to identify their sensitivity according to the modifications in the droop gain of the reactive power. Henceforth stability analysis of voltage is investigated.

A practice of proper reactive power sharing and to boost the microgrid behaviour exclusively in the island operation was done through the virtual impedance control system. This idea was put forth by Jinwei He [23]. Primarily it was targeted on the optimization of reactive power sharing via virtual impedance regulation. Subsequently, it exhibits the compensation of harmonic voltage at the PCC as well as perfect harmonic load sharing. This was accomplished by adjusting the DG unit corresponding to the harmonic impedances. Furthermore, the harmonic impedance and the virtual impedance were instrumented without having interference among them and thus it avoids the fundamental and the harmonic component drawn from the local controller of the DG unit. This was made possible by the resonant voltage controller adjustable arrangement.

A novel attempt from Christopher N. Rowe [22], reveals an enhancement in the stability of microgrid with the help of Arctan Power-Frequency Droop. They mainly aimed to enhance the small signal stability of the inverters in islanded microgrid via Arctan gradient algorithm. The Arctan droop pattern projected in this work eliminates the constant P-F droop slope also swaps it by an Arctan gradient algorithm. This theory was valued on the basis of system behavior accompanied by the target of voltage and frequency regulation. Simultaneously appropriate reactive power sharing among DGs was valued. Furthermore, the microgrid internal control was concentrated beneath the assumption that no infinite bus acts as a microgrid buffer. He tried the simulation in SABER package to find the operational values of steady state for Arctan droop.

\section{Power Sharing Method Using Modified Conventional Droop Control}

\subsection{P-V \& Q-F Droop Control}

The idea of proper power sharing in an inverter coupled with microgrid by means of P-V \& Q-V droop control was given by Qing-Chang Zhong and Charles K [28], [5]. In this technique, the main aim was to obtain power sharing as well as voltage and frequency regulation. Though their aim was similar but they differ from their control strategy.

By using droop control plan, Qing-Chang Zhong [28] executes load sharing by inverters operating in parallel. The constraints in conventional droop control i.e., the necessity to maintain the same per unit impedance and voltage set points of the inverter for proper power sharing. In this work, these constraints are overcome by the introduction of robust droop controller. This control scheme is robust to the various constraints like mismatches in components, numerical faults, disorders and noises. In this work, load voltage is regulated and hence droop effect was reduced. Also the voltage drop is compensated. Here, auxiliary control used to achieve rated values of deviating voltage and frequency. This tends to delay in measurement as well as a communication channel which causes slow response. This difficulty can be solvable with an integrated controller, so error in power sharing was quantitatively surveyed.

In addition, Charles K. [5] displays Voltage-Power droop control technique to maintain the voltage and frequency of microgrid in order to match the voltage and frequency coefficients by sharing a real and 
reactive load. Multiple VPD/FQB controllers combine and regulate the voltage of the microgrid by sharing a common power to a predefined ratio proportionately. It is achieved by drooping the voltage reference of every controller on d-axis current versus its real power output. Same way, the frequency reference of every controller on q-axis current is boosted and multiple VPD/FQB controllers combine regulate the microgrid frequency by sharing a reactive load to a predefined ratio proportionately. This method executes multiple VSC's works in parallel in VSC fed microgrid.

By using P-V and Q-F droop control, the active load can be controlled in island microgrids. These factors are briefly handled by Tine L. Vandoorn [27] and his main aim is to increase capability of islanded microgrid and thus it simplifies the line losses through demand dispatch method. Active load control is activated by the rms voltage of microgrid. This is qualified by the combination of $\mathrm{P}-\mathrm{V}$ droop control and voltage droops. For a low voltage microgrid, the Active load control is altered to the low consumption mode. For high voltage microgrid, it is altered to high consumption mode. This makes the Active load control more advantageous in reliability concerns. Vg-Vdc droop control united with Pdc-Vg is used for the control of active power. Also Q/F droop control theory demonstrates that, by altering the droops, reactive power can be regulated among the DG units. This reveals the reactive powers are shared properly.

\subsection{P-V Droop Control}

The independent operation of Hybrid Microgrid with P-V droop control is briefly shown in Poh Chiang Loh, Ding Li [29], [30] strategy. In their views, the active power was shared properly in hybrid microgrid by using the interlinked converters. It is a challenge of interlinking dc grid with ac grid through converters. This is not an easy thing in island operation.

In [29], the author concentrated on active power sharing and regulating DC voltage and AC frequency. The center of activity of AC grid is P-F droop control whereas P-V droop control for DC grid. AC-DC microgrid includes many subgrids which are combined by interlinking converters. The active powers are governed through equalizing the $\mathrm{DC}$ voltage and $\mathrm{AC}$ frequency. In order to share the power among the DGs proportionally to their power ratings, a new droop control scheme was introduced in an independent hybrid microgrid. The amounts of power transfer among the subgrids are forced by proportional power sharing in the entire hybrid microgrid converters.

Poh Chiang Loh also extended his research [29] by concerning the charging and discharging of storage devices [30]. The interconnected converters are formed by linking dc-dc boost converter with dc-ac inverter. This permits the voltage of dc-ac grid within the limit. It is verified that the accuracy of proportional active power sharing is not affected while charging and discharging of energy storage at the DC link of the converter through experimental results. PI controller is modeled to neutralize the voltage and frequency error. Also it is proved that proportional active power sharing is because of ratings not by sources in the hybrid microgrid.

In the perception of power sharing and voltage control, a modified droop control ie., P-V \& Vg-Vdc droop control was introduced by Tine L. Vandoorn and Bart Meersman [31], [33]

Tine L. Vandoorn [31], introduced a Smart transformer (ST) in order to maintain active power sharing among microgrids. This smart transformer (ST) works on an analogous control strategy to control the power sharing among microgrids and utility grid. By this effect the microgrid can change according to ST even if it is not communicated. By the introduction of ST, the voltage of all the terminals was increased and hence there is no necessity to alter power control method.

Whereas, [33] aimed to proven about the noble outcome in the issues like transient stability and power sharing. The stability and power sharing was obtained without communication between interunit. This causes beneficial concerning reliability and it attains grouping of renewable energy sources. By using $\mathrm{Vg}-$ $\mathrm{Vdc}$ and $\mathrm{P}-\mathrm{V}$ droop control techniques, a better power balancing is obtained. The voltage limit can be adjusted by using an output power of DG unit.

Tine L. Vandoorn and Jeroen D. M. De Kooning [32], explained the programmed power sharing alteration with $\mathrm{P}-\mathrm{V}$ droop control in $\mathrm{LV}$ resistive microgrids. The bondage between $\mathrm{P}$ and $\mathrm{V}$ are usual thing when considering resistive networks and so P-V droop control perform effectively. This strategy explains the natural power sharing by which line losses are simplified in whole system thus the efficiency increased subsequently. P-V and P-F droop control can be differentiated by changes in the power-sharing alteration and losses in the line.

\subsection{V-F Droop Control}


Mohammad N. Marwali's et al. [34] detailed study illustrates about the load sharing controller stability analysis by using V-F control for the distributed generation. The foremost aim was to interrogate about the paralleled DGs stableness in a stand-alone AC power supply mode. Also the small-signal stability was briefly analyzed with combinational droop as well as average power scheme for load sharing of manifold DGs. This says that the control system stability was predicted precisely by using the small - signal model.

Optimization of reactive power sharing in island operation through programmed virtual impedance by means of V-F droop control was put forth by Jinwei He et al. [35]. It was primarily aimed at the proportional real and reactive power sharing to the rated power of DG unit. A reactive power sharing technique was introduced for islanding microgrids and new power control technique was introduced to index load sharing deviation in island microgrids. In order to send harmonic voltage signals at PCC to the local DG controller from central controllers, a low bandwidth communication scheme was proposed. Also the DG unit feeder impedance can be found by this communication proposal. On controlling the virtual impedance, the reactive load was shared among parallel DG units with accurate proportion. Virtual impedance was converging on frequency, so the harmonic current flow was not affected. It also concerned about accurate reactive power sharing.

\subsection{Adaptive Transient Droop Control}

A droop control modified with adaptive gain was initiated by Yasser Abdel-Rady et al. [36] to investigate the decentralized droop controller in order to save the power sharing stability of inverters in parallel among DG microgrids. Their chief aim was to get stable and healthy power sharing act among paralleled inverter system. This technique involves both static and adaptive transient droop characteristics. This droop controller has 2 DOF tuneable controllers; by using this dynamic performance of power sharing can be altered. It doesn't affect the static droop gain while damping the oscillations in the power sharing controller. This technique stores the dynamics and stability of every inverter unit in different loading terms. Finally this proposal helps to preserve stability and reliability.

\section{5. $\quad$ SoC Based Droop Control}

A new methodology was put forth by Xiaonan Lu et al. [37] for dynamic power sharing on the basis of SoC (state-of-charge). In this work, the AC-bus voltage restoration with the help of SoC-based droop control technique was explained. The main aim of this proposal is to eradicate the problem of sharing power in the distributed energy storage system. The energy storage unit with higher SoC generates more active power than the lower Soc. By the SoC-based droop control, the frequency deviates from its nominal value. So an auxiliary control was introduced to restore the frequency and amplitude of the AC bus voltage. With this effect, the frequency and amplitude of the PCC voltage were also restored.

\subsection{Angle Droop Control}

Focusing on Rural distributed generation, Ritwik Majumder et al. [38], investigated the droop control of converters coupled with microsources. This was with the help of angle droop control. The signal from global positioning system controls the angle droop. Their compilation includes the aim of satisfactory power sharing under a weak system conditions and high resistive network. They discussed two control methods where the first one shares power between DGs with no communication. However the second one is less communication based output feedback controller. With this technique, the power sharing was proper and economical with the web based communication.

\subsection{Q-V Dot Droop Control}

A novel droop control scheme for Distributed Energy Resource (DER) interlinked by means of Converters was exposed by Chia-Tse Lee et al. [39], [40] in an island operation of microgrid. In the views of their compilation, the main purpose was to eradicate the effect of line impedance of each DIC (DERs interface converters) and to rise the reactive power sharing. In order to improve reactive power sharing in DIC's, QVdot droop control technique used, in which Vdot denotes the rate of change of voltage magnitude V. To make the Voltage magnitude in steady, a Vdot restoration technique was introduced. To get the desired 
reactive power sharing, DIC's voltage has to vary. This technique helps to overcome the effect of line impedance of every DIC. To compare power sharing results with general Q-V droop control and to evaluate the discussed Q-V dot droop control technique, a DIC test bench was made.

\section{Power Sharing Method Using Wireless Sensor Networks}

The influence of Wireless Sensor Networks in DG for the power sharing as well as control methods was displayed by Ritwik Majumder et al. [41]. For the optimization of power sharing it empowers reliable communication in DGs. In order to confirm reliable communication among the sensor nodes/DG and control center, the FRL (Find Reliable Link) scheme was suggested. This FRL scheme involves in the reduction of delay in end-to-end as well as it boost the packet success ratio. In addition this scheme involves when a node failure was occurred or else when the message was lost wholly owing to an obstacle located in the middle of the sender and the receiver.

\section{Conclusion}

Recently, the techniques based on control strategies in power sharing constraints have impressed the scholars. Much effort has been put forth to present a systematic review on the above mentioned issue. This review is about the challenges on power sharing difficulties and how to overcome the constraints when manifold DGs are linked to a microgrid via power electronic converter. A survey has been gone through elaborately. Various droop control techniques are highlighted in the literature that targeted on inadequacies of power sharing. This survey demonstrates the thirst of the researchers in the issues like active and reactive power sharing, voltage and frequency regulation. In the researcher's perception, this literature survey will be valued as a reference to work in the area of droop control application to meet the challenges of the power sharing issues in microgrid.

\section{References}

[1] Y. A. R. I. Mohamed and E. F. El-Saadany, "Adaptive decentralized droop controller to preserve power sharing stability of paralleled inverters in distributed generation microgrids," IEEE Transactions on Power Electronics, vol. 23, no. 6, pp. 2806-2816, 2008.

[2] K.-L. Nguyen, D.-J. Won, S.-J. Ahn, and I.-Y. Chung, "Power sharing method for a grid connected microgrid with multiple distributed generators," Journal of Electrical Engineering \& Technology, vol. 7, no. 4, pp. 459-467, 2012.

[3] H. Jiayi, J. Chuanwen, and X. Rong, "A review on distributed energy resources and microgrid," Renewable Sustainable Energy Rev., vol. 12, no. 9, pp. 2472-2483, 2008.

[4] P. Piagi and R. H. Lasseter, "Microgrid: A conceptual solution," Power Electronics Specialists Conference, vol. 6, pp. 4285-4290, 2004.

[5] C. K. Sao and P. W. Lehn, "Control and power management of converter fed microgrids," IEEE Transactions on Power System, vol. 23, no. 3, pp. 1088-1098, 2008.

[6] M. Griffiths and C. Coates, "Modelling and performance of low voltage distributed generation systems," in Proc. 6 th AUPEC Conference, Australia, 2006, pp. 10-13.

[7] PSERCR. H. Lasseter, "Control and design of microgrid components," Final Project Reports. [Online]. $\quad$ Available: http://www.pserc.org/cgipserc/getbig/publicatio/reports/2006report/lasseter_microgridcontrol_fin al_project_report.pdf

[8] P. Piagi and R. H. Lasseter, "Autonomous control of microgrids," in Proc. Power Engineering Society General Meeting, Montreal, QC, Canada, 2006, pp. 8.

[9] J. M. Guerrero, L. G. de Vicuna, J. Matas, M. Castilla, and J. Miret, "A wireless controller to enhance dynamic performance of parallel inverters in distributed generation systems," IEEE Transaction on Power Electron, vol. 19 , no. 5, pp. 1205-1213, September 2004.

[10] J. M. Guerrero, J. Matas, L. G. de Vicuna, M. Castilla, and J. Miret, "Decentralized control for parallel operation of distributed generation inverters using resistive output impedance," IEEE Transaction Ind. Electron., vol. 54, no. 2, pp. 994-1004, April 2007. 
[11] H. Liang, B. J. Choi, W. Zhuang, and X. Shen, "Decentralized inverter control in microgrids based on power sharing information through wireless communications," in Proc. Global Communications Conference (GLOBECOM) IEEE, 2012, pp. 5148-5153.

[12] J.-H. Choi, "Power sharing and frequency control of an autonomous microgrid considering the dynamic characteristics of distributed generations," The Journal of International Council on Electrical Engineering, wol. 2, no. 1, pp. 39-44, 2012.

[13] S.-J. Ahn, J.-W. Park, I.-Y. Chung, S.-I. Moon, S.-H. Kang, and S.-R. Nam, "Power-sharing method of multiple distributed generators considering control modes and configurations of a microgrid," IEEE Transaction on Power Delivery, vol. 25, no. 3, pp. 2007-2016, July 2010.

[14] J. He and Y. W. Li, "An enhanced microgrid load demand sharing strategy," IEEE Transaction on Power Electronics, vol. 27, no. 9, pp. 3984-3995, September 2012.

[15] F. Shahnia, R. Majumder, A. Ghosh, G. Ledwich, and F. Zare, "Operation and control of a hybrid microgrid containing unbalanced and nonlinear loads," Electric Power Systems Research, vol. 80, no. 8, pp. 954-965, 2010.

[16] R. Majumder, B. Chaudhuri, A. Ghosh, G. Ledwich, and F. Zare, "Improvement of stability and load sharing in an autonomous microgrid using supplementary droop control loop," IEEE Transaction on Power Systems, vol. 25, no. 2, pp. 796-808, May 2010.

[17] M. Kohansal, G. B. Gharehpetian, M. Abedi, and M. J. Sanjari, "Droop controller limitation for voltage stability in islanded microgrid," submitted for publication.

[18] F. Katiraei and M. R. Iravani, "Power management strategies for a microgrid with multiple distributed generation units," IEEE Transaction on Power Systems, vol. 21, no. 4, pp. 1821-1831, November 2006.

[19] J. Schiera, R. Ortegab, A. Astolc, J. Raischd, and T. Sezi, "Conditions for stability of droop-controlled inverter-based microgrids," submitted for publication.

[20] F. Razavi, R. Torani, I. Askarian, A. Asgharizadeh, and N. Masoomi, "Optimal design of islanded microgrid using genetic algorithm," presented at International Conference on Genetic and Evolutionary Methods (GEM'12), 2012.

[21] Q. Shafiee, J. C. Vasquez, and J. M. Guerrero, "Distributed secondary control for islanded microgridsA networked control systems approach," in Proc. of IECON 38th Annual Conference on IEEE Industrial Electronics Society, 2012, pp. 5637-5642.

[22] C. N. Rowe, T. J. Summers, and R. E. Betz, "Arctan power frequency droop for power electronics dominated microgrids," IEEE Transactions on Power Electronics, vol. 28, no. 8, pp. 3747-3759, Aug. 2013.

[23] J. He, Y. Li, J. Guerrero, F. Blaabjerg, and J. Vasquez, "An islanding microgrid power sharing approach using enhanced virtual impedance control scheme," IEEE Transaction on Power Electronics, vol. 28, no. 11, pp. 5272-5282, November 2013.

[24] II-Yop Chung, W. Liu, D. A. Cartes, E. G. Collins, and S.-I. Moon, "Control methods of inverterinterfaced distributed generators in a microgrid system," IEEE Transactions on Industry Applications, vol. 46, no. 3, pp. 1078-1088, May 2010.

[25] A. Micallef, M. Apap, C. Spiteri-Staines, and J. M. Guerrero, "Secondary control for reactive power sharing in droop-controlled islanded microgrids," In Industrial Electronics (ISIE), 2012 IEEE International Symposium on, 2012, pp. 1627-1633.

[26] X. Zhao-xia and F. Hong-wei, "Impacts of Pf \& QV droop control on microgrids transient stability," Physics Procedia, vol. 24, pp. 276-282, 2012.

[27] T. L. Vandoorn, B. Renders, L. Degroote, B. Meersman, and L. Vandevelde, "Active load control in islanded microgrids based on the grid voltage," IEEE Transactions on Smart Grid, vol. 2, no. 1, pp. 139151, March 2011.

[28] Q. C. Zhong, "Robust droop controller for accurate proportional load sharing among inverters operated in parallel," presented at the 18th IF AC W orld Congress, Milano, Italy, Sept. 2011.

[29] P. C. Loh and F. Blaabjerg, "Autonomous operation of hybrid microgrid with AC and DC Subgrids," IEEE Transactions On Power Electronics, vol. 28, no. 5, pp. 2214-2223, May 2013.

[30] P. C. Loh, D. Li, and F. Blaabjerg, "Autonomous control of interlinking converter with energy storage in hybrid AC-DC microgrid," IEEE Transactions On Industry Applications, vol. 49, no. 3, pp. 1374-1382, June 2013.

[31] T. Vandoorn, J. De Kooning, B. Meersman, J. Guerrero, and L. Vandevelde, "Voltage-based control of a smart transformer in a microgrid," IEEE Transactions on Industrial Electronics, vol. 60, no. 4, pp. 1291-1305, April 2013. 
[32] T. Vandoorn, B. Meersman, J. De Kooning, J. Guerrero, and L. Vandevelde, "Automatic power sharing modification of $\mathrm{P} / \mathrm{V}$ droop controllers in low-voltage resistive microgrids," IEEE Transactions on Power Delivery, vol. 2, no. 4, pp. 2318-2325, October 2012.

[33] T. L. Vandoorn, B. Meersman, L. Degroote, B. Renders, and L. Vandevelde, "A control strategy for islanded microgrids with dc-link voltage control," IEEE Transactions on Power Delivery, vol. 26, no. 2, pp. 703-713, 2011.

[34] C.-T. Lee, C.-C. Chu, and P.-T. Cheng, "A new droop control method for the autonomous operation of distributed energy resource interface converters," IEEE Transactions on Power Electronics, vol. 28, no. 4, pp. 1980-1993, April 2013.

[35] J. He, Y. W. Li, J. M. Guerrero, J. C. Vasquez, and F. Blaabjerg, "An islanding microgrid reactive power sharing scheme enhanced by programmed virtual impedances," in Proc. of Power Electronics for Distributed Generation Systems (PEDG), 3rd IEEE International Symposium, 2012, pp. 229-235.

[36] Y. A. R. I. Mohamed and E. F. El-Saadany, "Adaptive decentralized droop controller to preserve power sharing stability of paralleled inverters in distributed generation microgrids," IEEE Transactions on Power Electronics, vol. 23, no. 6, pp. 2806-2816, 2006.

[37] X. Lu, K. Sun, J. Guerrero, and L. Huang, "SoC-based dynamic power sharing method with AC-bus voltage restoration for microgrid applications," in Proceedings of $38^{\text {th }}$ IECON Annual Conference on IEEE Industrial Electronics Society, 2012, pp. 5677-5682.

[38] R. Majumder, G. Ledwich, A. Ghosh, S. Chakrabarti, and F. Zare, "Droop control of converterinterfaced microsources in rural distributed generation," IEEE Transactions on Power Delivery, vol. 25, no. 4, pp. 2768-2778, 2010.

[39] C.-T. Lee, C.-C. Chu, and P.-T. Cheng, "A new droop control method for the autonomous operation of distributed energy resource interface converters," in Energy Conversion Congress and Exposition (ECCE), IEEE, 2010, pp. 702-709.

[40] M. N. Marwali, J.-W. Jung, and A. Keyhani, "Stability analysis of load sharing control for distributed generation systems," IEEE Transactions on Energy Conversion, vol. 22, no. 3, pp. 737-745, 2007.

[41] R. Majumder, G. Bag, and K.-H. Kim, "Power sharing and control in distributed generation with wireless sensor networks," IEEE Transactions on Smart Grid, vol. 3, no. 2, pp. 618-634, 2012.

[42] Nandar, Cuk Supriyadi Ali, "Design of SMES controller to enhance stability of microgrid system taking coil size into consideration." Engineering Journal, Vol. 14, no. 4, 11-20, 2010.

[43] R. Zamora and A. K. Srivastava, "Controls for microgrids with storage: Review, challenges, and research needs," Renew. Sustain. Energy Rev., vol. 14, pp. 2009-2018, 2010.

[44] C.-X. Dou, D.-L. Liu, X.-B. Jia, and F. Zhao, "Management and control for smart microgrid based on hybrid control theory," Electric Power Components and Systems, vol. 39, no. 8, pp. 813-832, 2011.

[45] A. Llaria, O. Curea, J. Jimenez, and H. Camblong, "Survey on microgrids: Unplanned islanding and related inverter control techniques," Renewable Energy, vol. 36, no. 8, pp. 2052-2061, 2011. 
